\title{
Endosomal gene expression: a new indicator for prostate cancer patient prognosis?
}

\author{
Ian R.D. Johnson ${ }^{1}$, Emma J. Parkinson-Lawrence ${ }^{1}$, Helen Keegan ${ }^{2,3}$, Cathy D. \\ Spillane $^{3}$, Jacqui Barry-0'Crowley ${ }^{2}$, William R. Watson ${ }^{4}$, Stavros Selemidis ${ }^{5}$, Lisa \\ M. Butler ${ }^{6}$, John J. O'Leary ${ }^{2,3}$ and Doug A. Brooks ${ }^{1}$ \\ ${ }^{1}$ Mechanisms in Cell Biology and Disease Research Group, School of Pharmacy and Medical Sciences, Sansom Institute for \\ Health Research, University of South Australia, Adelaide, SA, Australia \\ 2 Department of Pathology, Coombe Women and Infants University Hospital, Dublin, Ireland \\ ${ }^{3}$ Department of Histopathology, Trinity College Dublin, Dublin, Ireland \\ ${ }^{4}$ UCD School of Medicine and Medical Science, Conway Institute of Biomolecular and Biomedical Research, University College \\ Dublin, Belfield, Dublin, Ireland \\ ${ }^{5}$ Infection and Immunity Program, Biomedicine Discovery Institute, Department of Pharmacology, Monash University, \\ Clayton, VIC, Australia \\ ${ }^{6}$ Prostate Cancer Research Group, School of Medicine and Freemasons Centre for Men's Health, University of Adelaide, \\ Adelaide, SA, Australia \\ Correspondence to: Doug A. Brooks, email: Doug.Brooks@unisa.edu.au \\ Keywords: prostate cancer, biomarkers, prognosis, endosomal gene expression, mRNA \\ Received: September 23, $2015 \quad$ Accepted: September 28, $2015 \quad$ Published: October 14, 2015
}

This is an open-access article distributed under the terms of the Creative Commons Attribution License, which permits unrestricted use, distribution, and reproduction in any medium, provided the original author and source are credited.

\section{ABSTRACT}

Prostate cancer continues to be a major cause of morbidity and mortality in men, but a method for accurate prognosis in these patients is yet to be developed. The recent discovery of altered endosomal biogenesis in prostate cancer has identified a fundamental change in the cell biology of this cancer, which holds great promise for the identification of novel biomarkers that can predict disease outcomes. Here we have identified significantly altered expression of endosomal genes in prostate cancer compared to non-malignant tissue in mRNA microarrays and confirmed these findings by qRT-PCR on fresh-frozen tissue. Importantly, we identified endosomal gene expression patterns that were predictive of patient outcomes. Two endosomal tri-gene signatures were identified from a previously published microarray cohort and had a significant capacity to stratify patient outcomes. The expression of APPL1, RAB5A, EEA1, PDCD6IP, NOX4 and SORT1 were altered in malignant patient tissue, when compared to indolent and normal prostate tissue. These findings support the initiation of a case-control study using larger cohorts of prostate tissue, with documented patient outcomes, to determine if different combinations of these new biomarkers can accurately predict disease status and clinical progression in prostate cancer patients.

\section{INTRODUCTION}

Prostate cancer is the second most commonly diagnosed cancer in males [1], and the incidence of this disease is predicted to double globally by 2030 (WCRF prostate cancer statistics; http://globocan.iarc.fr, accessed May 2014). More than 1.1 million new cases of prostate cancer are diagnosed each year and two thirds of these patients are from the Western world. The marked increase in the age adjusted incidence rate for prostate cancer has been partly attributed to the prostate specific antigen (PSA) test identifying men without clinical symptoms of the disease. Unfortunately, the PSA biomarker neither discriminates between patients who are at a higher risk of progressive disease/mortality and those who have a more favorable prognosis, nor can it adequately distinguish between prostate cancer and benign pathologies $[2,3]$. Therefore, there is a significant need for an effective 
method to accurately define the prognosis for prostate cancer patients.

The investigation of biomarker expression by microarray analysis in patient cohorts, in relation to known clinical parameters, can be used to develop methods for determining patient prognosis [4-6]. Consequently, gene expression profiles that compare prostate cancer to benign prostatic hyperplasia $(\mathrm{BPH})$, prostatic intraepithelial neoplasia (PIN) and normal prostate tissue have been generated from microarray data [4]. This approach has been utilized to identify the enzyme $\alpha$-methylacyl-CoA racemase $(A M A C R)$, which was highly expressed in prostate cancer and may have value as a prognostic marker for the disease [7]. However, prostate cancers display substantial inter- and intra-tumor heterogeneity and the altered expression of a single gene may not be predictive for the wider prostate cancer cell population. In addition, altered gene expression may reflect de-differentiation and progression of tumor growth; for example, $A M A C R$ is an androgen-regulated gene and exhibits variable expression upon androgen-deprivation therapies or androgenindependent disease progression [8]. Therefore, signatures incorporating multiple genes may be required to improve the accuracy of prostate cancer prognosis.

Commercial tests have recently been developed in an attempt to distinguish between aggressive prostate cancer and indolent disease (reviewed by Sartori \& Chan 2014 [9]). The Prolaris ${ }^{\circledR}$ test measures the expression of 46 genes involved in cell cycle progression [10], whilst the Oncotype DX ${ }^{\circledR}$ Prostate Cancer Test measures the expression of genes involved in stromal response, cellular organization, proliferation, basal epithelial function, androgen signaling and stress response [11]. While these tests have entered clinical practice in the USA, and alongside the current PSA blood test, can be used to aid in clinical decision making, they do not predict progression to castrate-resistant cancer or determine responses of cancer cells to therapy [12]. Prostate cancer mRNA microarrays were used in the development of these biomarkers, suggesting that this approach has the potential to identify clinically-relevant new prostate cancer biomarkers.

We recently reported that the biology of endosomes is markedly altered in prostate cancer cells [13, 14] and postulated that the expression of these genes might be predictive of disease progression in prostate cancer patients. Endosomes are essential organelles that are involved in cellular energy metabolism, cell division, intracellular signaling and degradation; and are known to have a role in cancer pathogenesis [15]. For example, endosomal cathepsins have previously been reported to be involved in the process of metastasis [16], presumably through their role in the degradation of extracellular matrix. The endosome system also has a specific capacity to respond to cellular and environmental change and may be altered as the cancer grows. We therefore hypothesized that endosome-related genes will be altered in prostate cancer and provide novel gene biomarkers for use in prostate cancer prognosis.

Here, we have investigated endosomal gene expression in multiple independent prostate cancer cohorts and developed two endosomal gene signatures that were predictive of patient outcome. We have also evaluated endosomal gene expression in fresh-frozen tissue sections from radical prostatectomies and demonstrated a capacity to distinguish indolent from aggressive tumors. This study provides evidence that endosomal genes can distinguish prostate cancer patient outcomes and predict disease progression, warranting further investigation of these findings in larger case-control studies.

\section{RESULTS}

\section{Altered endosome associated gene expression in the Tomlins microarray patient cohort}

The expression of APPL1 and EEA1 was significantly increased in primary prostate cancer when compared to non-malignant controls $(P \leq 0.05$; Figure 1). The expression of $R A B 5 A$ and $E E A 1$ was significantly reduced in metastatic prostate cancer when compared to primary prostate cancer tissue $(P \leq 0.05$; Figure 1$)$. The expression of $R A B 4 A$ was significantly decreased in primary prostate cancer when compared to PIN tissue $(P \leq 0.05$; Figure 1$)$ and there was a significant reduction of $R A B 4 A$ expression in metastatic prostate tissue when compared to both non-malignant prostate cancer $(P \leq$ $0.01)$ and PIN tissue $(P \leq 0.0001$; Figure 1$)$. PDCD6IP was significantly decreased in metastatic prostate tissue when compared with both primary cancer and PIN tissue $(P \leq 0.01)$. The expression of NOX4 was significantly increased in metastatic prostate tissue when compared with PIN $(P \leq 0.05)$. Acid ceramidase $(A S A H 1)$ expression was significantly increased in PIN when compared to nonmalignant and primary prostate cancer tissue $(P \leq 0.05)$, and was significantly reduced in metastatic tissue when compared to primary prostate cancer $(P \leq 0.01)$, PIN $(P \leq$ $0.0001)$, and non-malignant tissue $(P \leq 0.01)$. Cathepsin $\mathrm{B}(C T S B)$ expression was significantly reduced in both primary cancer tissue and metastatic cancer tissue when compared to non-malignant prostate tissue $(P \leq 0.01$; Figure 1).

\section{Endosome associated gene expression is associated with survival outcome in prostate cancer patients}

From the Glinsky cohort [18], patients were classified into two groups of relative high and low mRNA expression of endosome-related genes with an arbitrary cut-point between the two groups defined by $K$-means clustering. There was increased expression of the cation- 

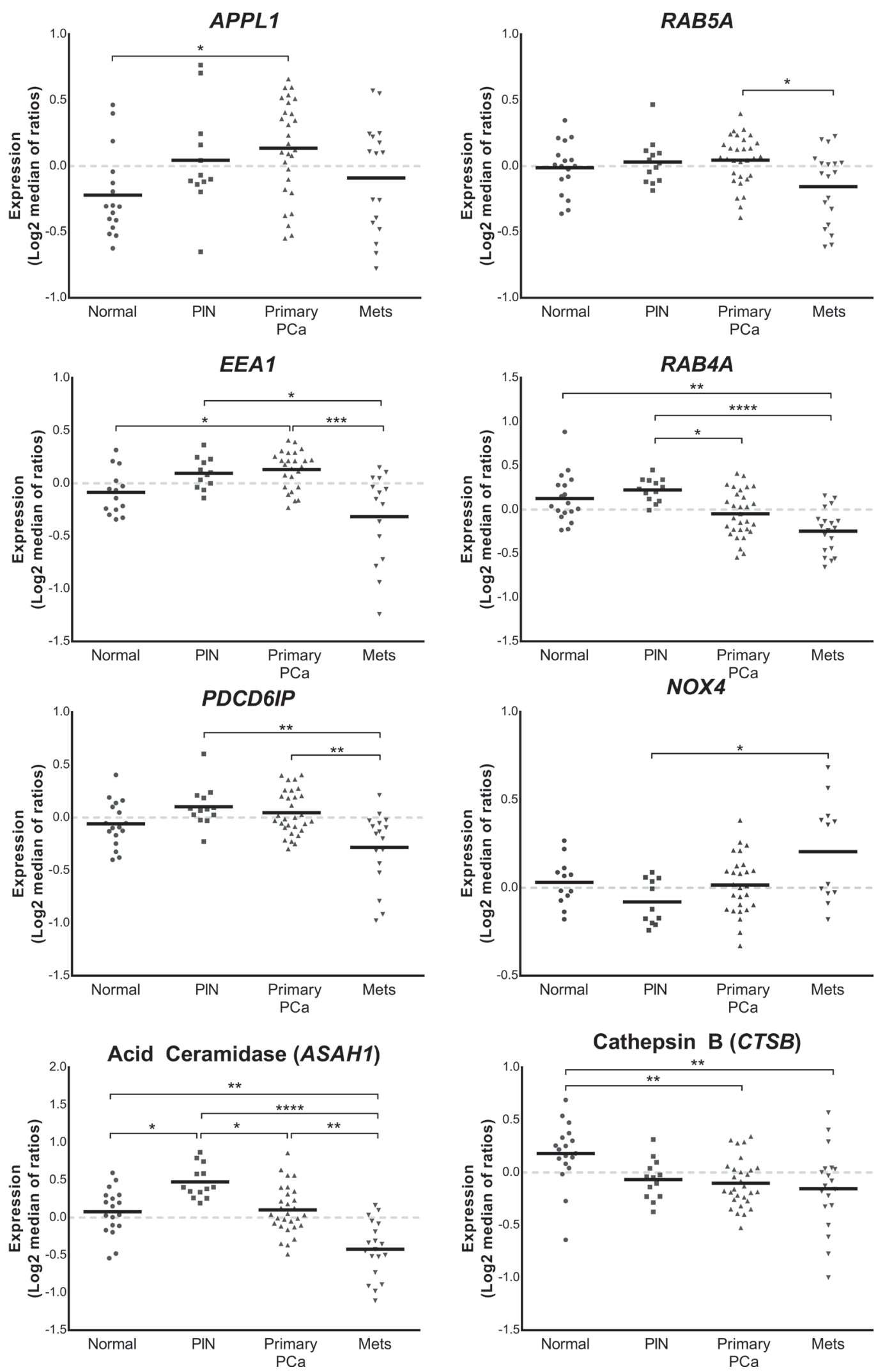

Figure 1: Vertical scatter plots of endosome-associated gene expression data from the Tomlins cohort [17]. Expression profiling data derived from the Chinnaiyan Human 20K Hs6 array of 18 non-malignant tissues, 13 prostatic intraepithelial neoplasias, 30 primary prostate cancer and 19 metastatic cancer tissue samples were quantitated to show relative amount of expression of lysosomalrelated genes. Statistical significance is represented by an asterisk $(* P \leq 0.05 ; * * P \leq 0.01 ; * * * P \leq 0.001 ; * * * * P \leq 0.0001)$. 
independent mannose 6-phosphate receptor $(I G F 2 R)$ in patients who had a greater risk of relapse $(P=0.007$; Figure 2). Clustering of high or low cathepsin B (CTSB) expression revealed patients with lower CTSB expression had significantly increased risk of biochemical recurrence $(P=0.0306)$. There was also a significant stratification of patients with Sortilin (SORT1) expression, with those patients who expressed greater amounts of SORT1 at an increased risk of relapse $(P=0.004)$. Myosin 1B $(M Y O 1 B)$
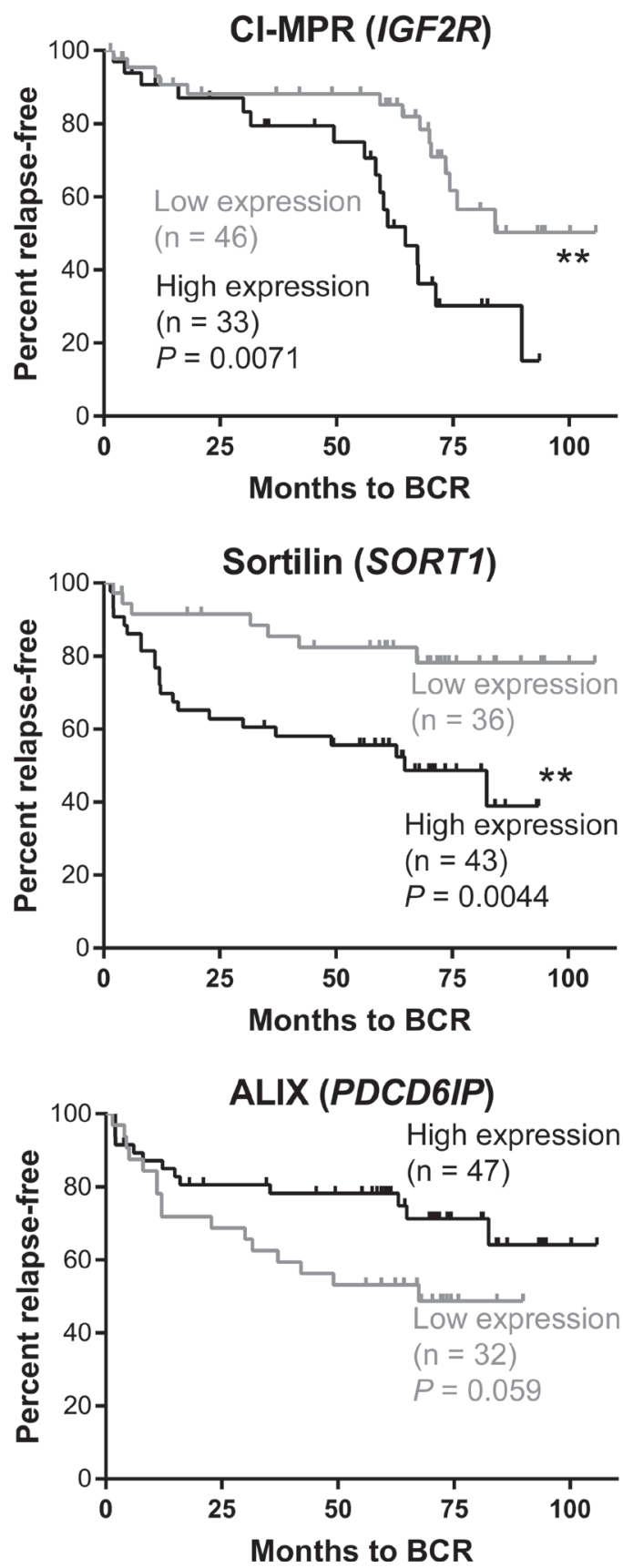

stratified patients at risk of recurrence $(P=0.03)$, with patients having increased expression being associated with a poorer prognosis. There was a trend for lower expression of ALIX (PDCD6IP) to stratify patients with increased prostate cancer recurrence $(P=0.059)$, and reduced expression of Syntaxin 12 (STX12) was also indicative of at-risk patients, with significant stratification $(P=0.001$; Figure 2).
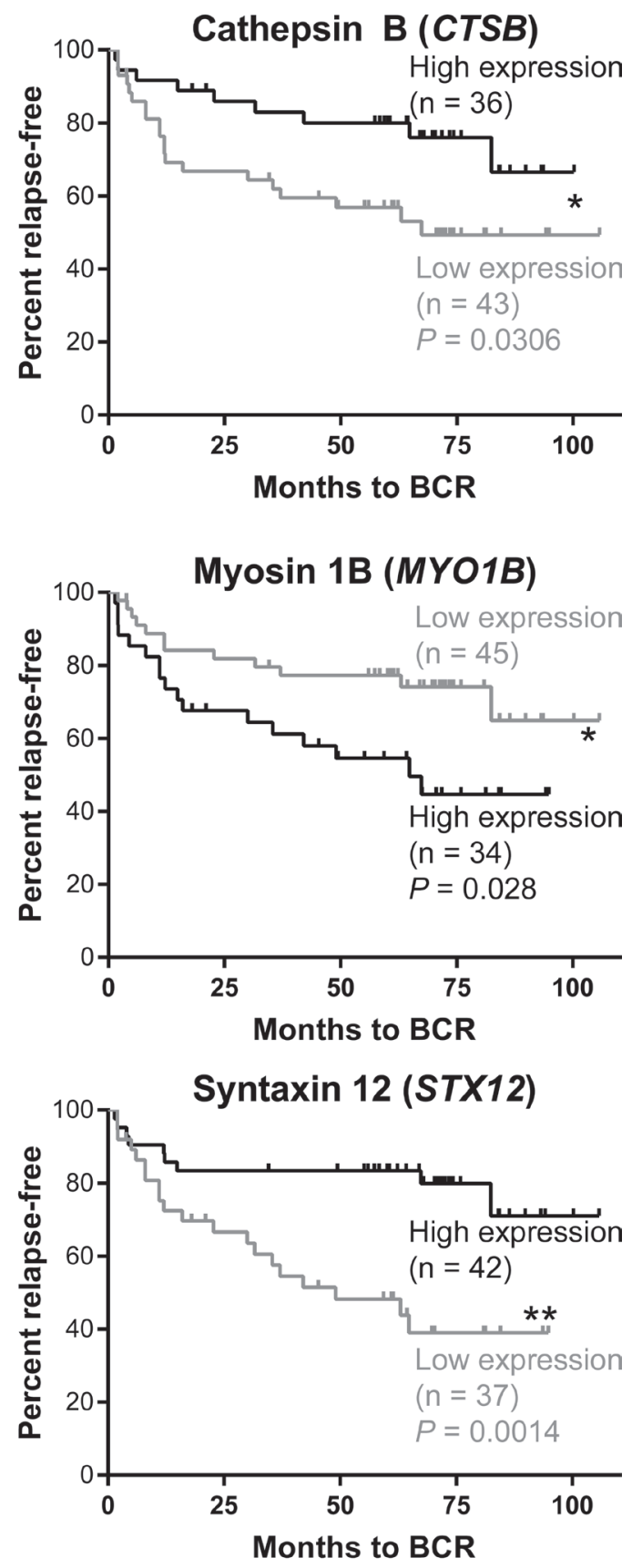

Figure 2: Kaplan-Meier analysis of endosomal genes and patient stratification based on biochemical recurrence (BCR). Patients from the Glinsky cohort [18] were stratified into two groups by $K$-means clustering based on amount (high - black line, low - grey line) of gene expression in prostate cancer samples. Analysis was performed using the Log-Rank test. IGF2R $(P=0.007), C T S B$ $(P=0.03)$, MYO1B $(P=0.03)$, SORT1 $(P=0.004)$ and STX12 $(P=0.001)$ differentiated patients at risk of relapse based on the amount of gene expression showed evidence of prognostic capacity. 


\section{Endosomal gene expression can predict clinical outcomes in prostate cancer patients with low amounts of PSA}

Analysis from the Tomlins cohort suggested that the mRNA expression of endosome-related genes was altered during disease progression, and that they might therefore have prognostic capacity. Of the patients in the Glinsky cohort that had pre-prostatectomy PSA levels of less than $10 \mathrm{ng} / \mathrm{mL}, 36.5 \%$ had biochemical failure at 100 months (data not shown). We postulated that the altered endosome gene expression in this patient group may indicate changes in cell biology that promote a more aggressive disease, and that this change could stratify patients at risk of recurrence, where the expression of PSA was low or borderline in blood samples.

Combinations of endosomal genes were analyzed to determine their potential for risk stratification, with a focus on genes related to functional endosome machinery in specific spatiotemporal compartments. Stratification of patients based on the expression of a RAB5A, APPL1 and $E E A 1$ tri-gene signature, using $K$-means clustering methodology, robustly separated patients into two groups with low or high expression of each of the three genes (Figure 3A). Kaplan-Meier survival analysis indicated that patients in the high expression group for this threegene signature were at significantly higher risk of biochemical recurrence when compared to those in the lower-expression group (HR 2.947, $P=0.0397,95 \%$ CI

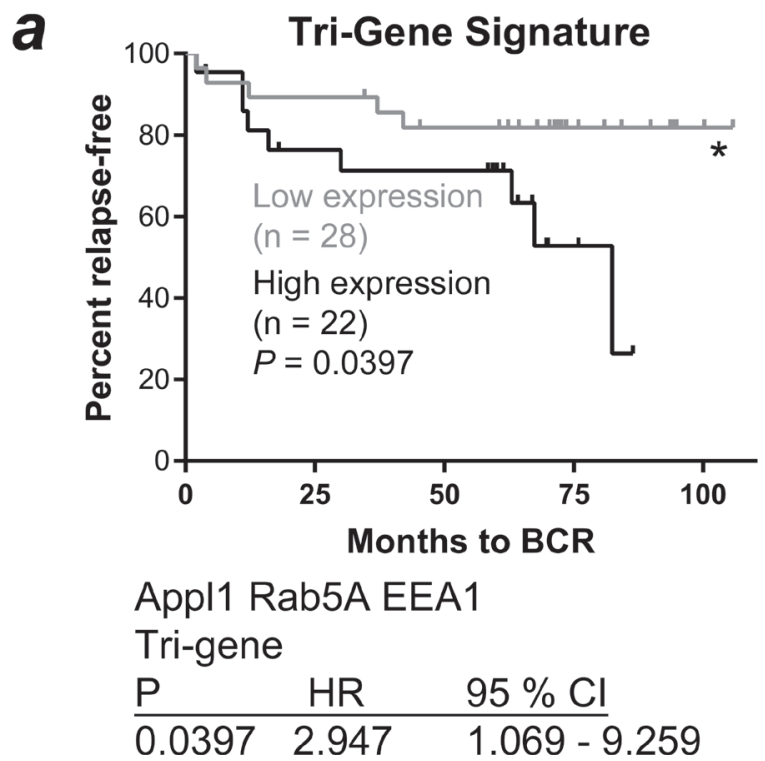

1.069 - 9.259; Figure 3A). Importantly, stratification of patients based on the expression of a combined MYO1B, PDCD6IP and STX12 tri-gene signature, using K means clustering, robustly separated patients into a low- and high-risk group that showed a greater stratification capacity than any of the single genes $(P=0.003$; HR $2.947, P=0.0397,95 \%$ CI $1.069-9.259$; Figure 3B). The high-risk group displayed lower expression of $M Y O 1 B$ and increased expression of both PDCD6IP and STX12.

\section{qRT-PCR analysis of fresh-frozen prostate tissue revealed significantly altered endosome-associated gene expression in aggressive prostate cancer}

qPCR analysis of endosome associated mRNA in fresh-frozen prostate cancer tissue demonstrated significantly increased expression of APPL1 in tissue from aggressive prostate cancer compared to nonmalignant prostate and indolent prostate cancer tissue $(P$ $\leq 0.01$; Figure 4). The expression of RAB5A and EEA1 were significantly increased in aggressive cancer tissue compared to indolent diseased tissue $(P \leq 0.05)$. The expression of NOX4 and SORT1 were also significantly increased in aggressive cancer tissue when compared with non-malignant $(P \leq 0.01)$ and indolent cancer tissue $(P$ $\leq 0.05)$. There was a significant reduction of PDCD6IP mRNA in indolent cancer tissue when compared to both non-malignant and aggressive prostate cancer tissue $(P \leq$ 0.05 respectively).

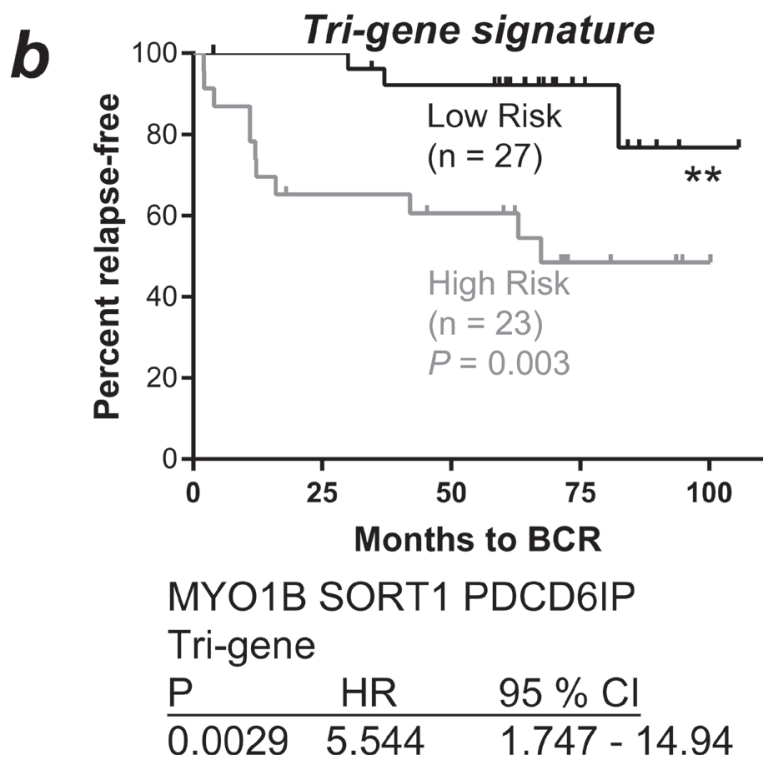

Figure 3: A. Kaplan-Meier survival analysis of a combined APPL1, RAB5A and EEA1 gene signature for cancer patients expressing $\leq$ $10 \mathrm{ng} / \mathrm{mL}$ PSA. Patients from the Glinsky cohort [18] expressing PSA $\leq 10 \mathrm{mg} / \mathrm{mL}$ were stratified into groups by $K$-means clustering based on RAB5A, APPL1 and EEA1 gene expression; the three-gene combined signature of APPL1, RAB5A and EEA1 stratified patients based on $\mathrm{BCR}(P \leq 0.0397$, Log-Rank test; high expression - black line, low expression - grey line). B. Kaplan-Meier survival analysis of $M Y O 1 B$, $P D C D 6 I P$ and $S T X 12$ expression and combined gene signature for cancer patients expressing $\leq 10 \mathrm{ng} / \mathrm{mL}$ PSA; the three-gene combined signature of MYO1B, PDCD6IP and STX12 stratified patients based on BCR $(P \leq 0.0029$, Log-Rank test; low risk - black line, high risk grey line). BCR: biochemical recurrence; HR: hazard ratio; CI: confidence interval. 


\section{DISCUSSION}

Prostate cancer is one of the most frequently diagnosed cancers in men, and a leading cause of cancer related deaths worldwide, particularly in the Western world [20, 21]. The PSA test is currently the gold-standard for prostate cancer detection, but is limited for specificity and has limited capacity for prognostic prediction [22]; consequently it cannot readily discriminate patients at higher risk of progressive disease or mortality from those who have a more favorable prognosis. Serum PSA results

APPL1

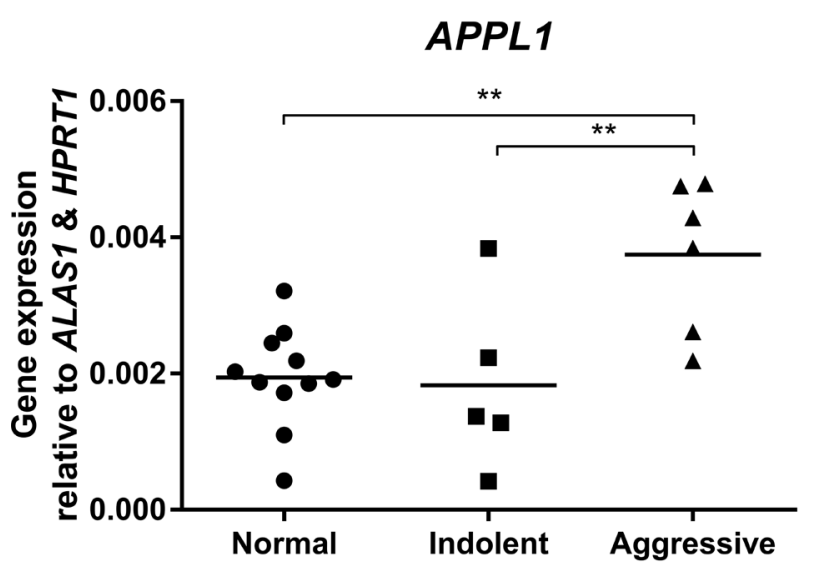

EEA1
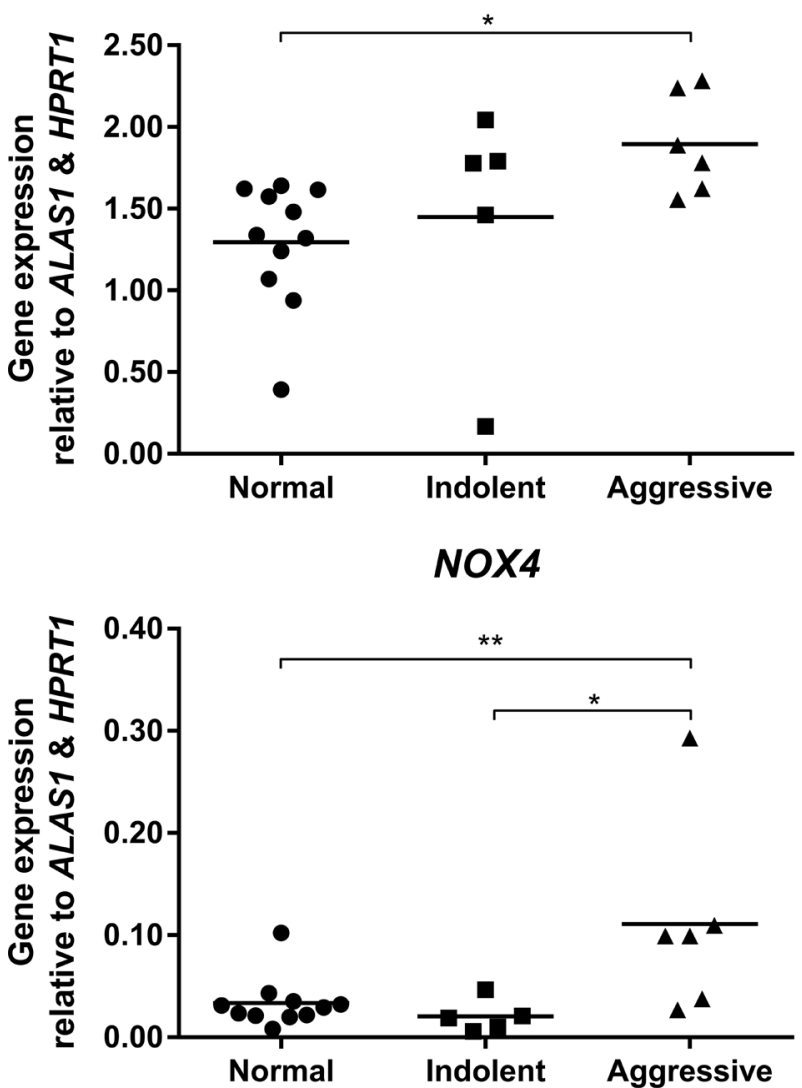

require contextual interpretation and judgment for each patient, however there are significant problems with specificity. The clinical decision-making process might be improved by the incorporation of multiple prostate cancer biomarkers, especially those that are associated with altered cancer cell biology.

Prognostic nomograms that incorporate continuous as well as categorical variables can potentially be improved by the integration of gene expression analysis and prognostic molecular signatures [23]. Our recent discovery of altered endosome biogenesis in prostate

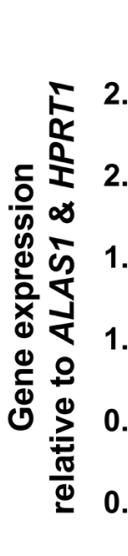

RAB5A

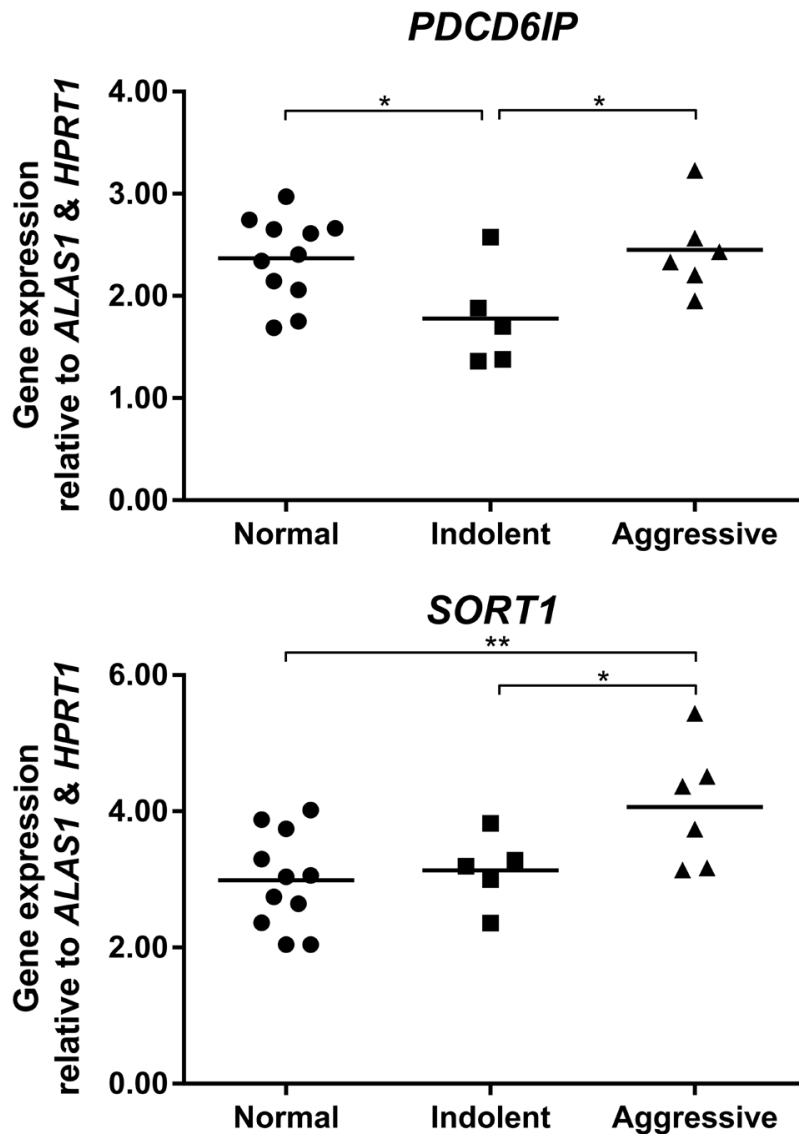

Figure 4: Vertical scatter plots of endosome-associated gene expression from qPCR mRNA analysis on non-malignant $(n=11)$, indolent $(n=5)$ and aggressive $(n=6)$ prostate cancer tissues from the Irish Prostate Cancer Research Consortium, Dublin, Ireland. Statistical significance is represented by an asterisk $(* P \leq 0.05 ; * * P \leq 0.01)$. 
cancer [14] suggests that the biologically relevant changes associated with these endosomal genes have the potential to improve the clinical decision-making process. There are an increasing number of commercial tests for biomarkers that complement the use of PSA (reviewed by Sartori \& Chan 2014 [9, 24]), but these tests still do not adequately provide an early accurate prognosis for prostate cancer; and coincidentally, have been discovered anecdotally or through biomarker screening rather than by association with a cell biological process. Gene expression may also be altered throughout the course of the cancer growth and differentiation; for example, $\alpha$ methylacyl CoA racemase is an androgen-regulated gene and exhibits variable expression upon androgendeprivation therapies or androgen-independent disease progression [8], requiring continued monitoring of these changes. The combined ratio of $P C A 3$ and $P C A$ expression in prostate cells extracted from urine, can indicate the likelihood of prostate cancer, even if biopsies return a negative result [25]; providing a rationale for the use of multiple biomarkers. The altered gene expression in these adjunct tests can elucidate changes in the biology of prostate cancer, such as $P C A 3$, which is associated with prostate cancer-cell survival and androgen receptor signaling [26]. This suggests that altered gene expression might need to be linked to functional cell biology to provide new avenues for the development of effective prognostic methodology. However, these new candidate genes can still be investigated in microarray cohorts to help retrospectively determine whether the biomarkers are informative of clinical outcome.

We recently reported that the biogenesis of endosomes is altered in prostate cancer, providing significant data on gene and protein expression in cultured prostate cells $[13,14]$. We found that the molecular machinery involved in early endosome biogenesis had altered expression in prostate cancer cells, and that the intracellular location of these compartments was altered, leading to dysregulation of proliferative signals. To establish that these novel cellular changes were clinically relevant, we confirmed here that the endosomal mRNA expression was altered in prostate cancer tissues and was indicative of disease progression; suggesting that these biomarkers might be suitable for prognosis in prostate cancer patients. Our novel gene signatures stratified patients into high and low-risk disease recurrence groups and demonstrated prognostic potential in those patients expressing low or borderline amounts of PSA.

The genes employed for these prognostic signatures are associated with separate populations of early-endosome compartments, thus reflecting that the early endosome population as a whole is disturbed in prostate cancer, rather than one specific compartment, complementing the prior discovery of altered endosome biogenesis in prostate cancer [14]. Altered endosomallysosomal biology and the associated changes in gene expression that occur during cancer development may be valuable prognostic indicators; for example, in breast cancer the increased expression of the endosomal protein acid ceramidase $(A S A H 1)$ has been associated with improved outcomes [27]. Interestingly, $A S A H 1$ maps to chromosome $8 \mathrm{p} 22$, which is frequently deleted in prostate cancer patients [28, 29]. Endosomal CTSB (cathepsin B) is also found on chromosome $8 \mathrm{p} 22$ and the down regulation of $C T S B$ in metastatic tissue may also relate to these chromosomal break points. The reductions in both $A S A H 1$ and $C T S B$ expression in metastatic tissue may therefore be related to this deletion and furthermore is linked to the altered biogenesis of endosomes as these organelles are used to transport both of the latter enzymes.

The changes in APPL1 (3p21), RAB5A (3p24) and EEA1 (12q22) and $R A B 4 A(1 \mathrm{q} 42)$ in PIN or primary cancer tissue compared to their expression in nonmalignant tissue may discriminate metastatic tissue and provide an avenue for monitoring disease progression. The changes in the expression of endosomal genes observed in the Tomlins cohort suggested that they may be involved in the initiation or promotion of pre-malignant cell survival, and that sustained expression is not essential for the continued function of the malignant cell. Indeed, the transgenic adenocarcinoma of the mouse prostate (TRAMP) model of prostate cancer displays elevated gene expression in PIN tissue, which is later reduced in tumors [30]. The wide variation of gene expression changes in the metastatic tissue of the Tomlins cohort, when compared with primary cancer or PIN tissue may reflect the degree of dysfunction in these cells, which in turn contributed to the metastatic cascade. Further study with matched normal and disease samples would ascertain the extent of expression changes for each gene, for the development of a more refined method to predict disease progression.

Microarrays provide only a 'snapshot' of a cell population and there is potential for mRNA to be secreted prior to, or post protein-synthesis [31-33], complicating the interpretation of gene expression data. This regulation of the functional gene is equally critical to cancer cell biology and in the case of cathepsin B, the promotion of cancer metastasis through the hydrolytic activity of this enzyme [16]. Thus the incorporation of protein biomarkers and or functional enzymes, analyzed independently of their respective gene expression, may offer further insight into specific cell biology changes and provide more accurate prognosis.

We have noted the limitations of microarray gene expression analysis and consequently performed qPCR analysis of fresh-frozen prostate tissue to confirm our findings on endosomal gene expression. It should be recognized that the Glinsky cohort does not provide information on the gene expression in normal tissue from the cancer patients, or healthy individuals that have increased PSA independent of cancer occurrence, thus the current gene signatures are indicative of prognostic 
potential rather than necessarily stratifying healthy individuals from those with prostate cancer. Furthermore, incorporating the Gleason scores from the Tomlins and Glinsky cohorts have been precluded from this study, due to the variability in the prognosis from this scoring system [34]. However, a dedicated case-control study utilizing these existing biomarkers and pathology tests, together with the endosomal biomarkers identified here, may result in an improved prognostic assay for prostate cancer.

The significant changes to APPL1, RAB5A and $E E A 1$ gene expression in prostate tissue from aggressive disease compared to normal tissue or indolent-disease tissue indicates that these biomarkers have significant potential to monitor disease progression and to stratifying patients at risk of progressive and or metastatic disease. They also have important biological relevance that could provide a therapeutic target, since alterations in endosome biogenesis can result in significantly altered intracellular cell signaling, result in increased cell proliferation and in combination with NOX4 the expression promote inflammation and angiogenesis [35].

In summary, endosomal gene expression and specific gene signatures have the potential to prediction disease progression in prostate cancer patients and this may lead to improved treatment outcomes. Multivariate risk analysis and stratification of patients into low- and high-risk groups may provide a more individualized approach to prostate cancer patient management, reducing the amount of over-treatment [36]. Active-surveillance is often used for patients deemed to have low-risk prostate cancer (e.g. clinical category T1c, Gleason score $\leq 6$, and $\mathrm{PSA} \leq 10 \mathrm{ng} / \mathrm{mL}$ ), but older men are at an increased risk of mortality from prostate cancer, despite low pathology scores and low PSA serum concentrations [37]. Examination of the survival rates for prostate cancer patients expressing low PSA, which is typical of an active surveillance group, reveals some prostate cancers that are aggressive and that result in more rapid recurrence. In the current study, quantitation of gene signatures for the early endosomal genes $A P P L 1, E E A 1$ and $R A B 5 A$, and analysis of the endosomal genes $M Y O 1 B, P D C D 6 I P$ and $S T X 12$ in prostate cancer patients expressing PSA $\leq 10$ $\mathrm{ng} / \mathrm{mL}$, led to patient stratification into high and low-risk recurrence groups. These findings justify further clinical investigation of these biomarkers and gene signatures in a dedicated case-control study. Recognizing that prostate cancer patients have altered endosomal gene expression is a significant step towards the development of new, biology-driven biomarkers that could provide accurate prognosis for this important disease.

\section{MATERIALS AND METHODS}

\section{Patient cohorts}

The Tomlins [17] cohort was chosen for its curation of multiple disease stages of prostate cancer. Analysis of tissue samples from this cohort was previously performed using the Chinnaiyan Human 20K Hs6 array [17] and was retrieved from NCBI GEO (accession number GSE6099). It is comprised of 18 non-malignant tissues, 13 prostatic intraepithelial neoplasia's (PIN), 30 primary prostate cancers obtained from radical prostatectomies and 19 metastatic cancer tissue samples were obtained from hormone refractory metastases in the liver, lung or lymph tissue [17]. The Glinsky cohort [18] was obtained from patients who had been treated by radical prostatectomy at Memorial Sloan-Kettering Cancer Center (MSKCC) and was composed of 79 malignant prostate tissues, with clinical follow-up data over 8 years, which was used to assess prostate cancer biochemical recurrence. This cohort comprised 29 patients with biochemical recurrence as defined by a PSA concentration $\geq 0.2 \mathrm{ng} /$ $\mathrm{mL}$ and 50 patients with no disease progression. Samples in this cohort had been examined histologically using H\&E-stained cryostat sections, non-neoplastic tissues removed from tumor samples and cells of interest manually dissected from the frozen block, with other tissues trimmed away [18]. Fresh-frozen prostate samples were provided by the Irish Prostate Cancer Research Consortium (PCRC) Bioresource, Conway Institute of Biomolecular and Biomedical Research, University College Dublin, Ireland; following approval from the Mater Misericordiae University Hospital ethics committee and written informed consent from all patients. The curated samples were selected based on the disease status of "aggressive" or "indolent". Aggressive disease samples $(\mathrm{n}=6)$ were selected from patients with biochemical recurrence (Median 27 months) and the median Gleason score was 7 with evidence of capsular invasion. Indolent cancer samples $(n=5)$ were selected from patients with no biochemical recurrence and had a median Gleason score of 5, with no evidence of capsular invasion. Matched non-malignant tissues $(\mathrm{n}=11)$ were also obtained from this cohort. Tissue histology was further confirmed by an expert pathologist upon re-section of each tissue specimen and subsequent H\&E staining. Based on the histology findings, a portion of prostate tissue, containing the highest tumor content, was cut from the specimen. These sub-samples were stored at -80 oC before RNA extraction.

\section{Quantitative RT-PCR}

Quantitative RT-PCR was performed on patient samples from the Irish PCRC cohort. RNA was extracted 
from non-malignant $(\mathrm{n}=11)$, indolent $(\mathrm{n}=5)$ and aggressive cancer tissues $(n=6)$. Reverse transcription of $500 \mathrm{ng}$ of total RNA was performed using a high-capacity cDNA Reverse Transcription Kit (Life Technologies Pty. Ltd., Cat\# 4368814). Real-time PCR was performed using $2 \mu \mathrm{L}$ cDNA for each of the following TaqMan ${ }^{\circledR}$ gene expression assays (Single Tube TaqMan ${ }^{\circledR}$ Gene Expression Assays Life Technologies); APPL1 (Hs00179382_m1), RAB5A (Hs00991290_m1), EEA1 (Hs00929215_m1), PDCD6IP (Hs00994346_m1), NOX4 (Hs00418356_m1), SORT1 (Hs00361760_m1) and TaqMan ${ }^{\circledR}$ Gene Expression Master Mix in a total volume of $10 \mu \mathrm{L}$. Three biological replicates were performed consisting of three technical replicates. Expression of each gene was normalized to an average of ALAS1 (Hs00963534_m1) and HPRT1 (Hs02800695_m1) reference genes and relative quantities calculated by $\bar{\Delta} \mathrm{Ct}$.

\section{Statistical analysis}

A Kruskal-Wallis test with Dunn's multiple comparison was performed to evaluate differences in gene expression between multiple groups in the Tomlins cohort. Data from the Glinsky cohort was analyzed using $K$-means clustering by Cluster 3.0 [19] to determine high and low gene expression groups. These groups were evaluated using Kaplan-Meier survival curves with differences determined using the Log-Rank (Mantel-Cox) test. The construction of tri-gene signatures also used $K$-means clustering methodology to cluster the expression values of the three genes from each tissue, resulting in two clusters representing high or low expression for each of the three genes. One-way ANOVA was performed to evaluate expression differences between non-malignant, indolent and aggressive prostate cancer tissues from the PCRC bioresource. All statistical tests were performed using GraphPad Prism 6.05 (GraphPad Software Inc., California, USA). Significant results showed greater than 95\% confidence $(P<0.05)$.

\section{ACKNOWLEDGMENTS}

This project was funded by a University of South Australia Presidents Scholarship, a University of South Australia Postgraduate Award, University of South Australia Research SA Seeding Funds, NHMRC development grant, Trevor Prescott Memorial Scholarship from the Freemasons' Foundation and Australian Research Council Future Fellowships for SS and LB (IDs FT1210876 and FT130101004). The Irish Prostate Cancer Research Consortium is supported by the Irish Cancer Society and the Health Research Board/Welcome Trust funded Dublin Centre for Clinical Research. We acknowledge the support of Dr. Amanda O'Neill in the identification and pulling of these tissue samples and the staff of the Histopathology Laboratory at Coombe Women and Infants University Hospital Dublin and the Friends of the Coombe for their support for this study.

\section{CONFLICTS OF INTEREST}

There is no conflict of interest.

\section{Editorial note}

This paper has been accepted based in part on peerreview conducted by another journal and the authors' response and revisions as well as expedited peer-review in Oncotarget.

\section{REFERENCES}

1. Siegel R, Naishadham D and Jemal A. Cancer statistics, 2013. CA: a cancer journal for clinicians. 2013; 63:11-30.

2. De Nunzio C, Kramer G, Marberger M, Montironi R, Nelson W, Schroder F, Sciarra A and Tubaro A. The controversial relationship between benign prostatic hyperplasia and prostate cancer: the role of inflammation. European Urology. 2011; 60:106-117.

3. Tarhan F, Orçun A, Küçükercan I, Çamursoy N and Kuyumcuoglu U. Effect of prostatic massage on serum complexed prostate-specific antigen levels. Urology. 2005; 66:1234-1238.

4. Aaltomaa S, Karja V, Lipponen P, Isotalo T, Kankkunen JP, Talja M and Mokka R. Reduced alpha- and beta-catenin expression predicts shortened survival in local prostate cancer. Anticancer Research. 2005; 25:4707-4712.

5. Markert EK, Mizuno H, Vazquez A and Levine AJ. Molecular classification of prostate cancer using curated expression signatures. Proceedings of the National Academy of Sciences of the United States of America. 2011; 108:21276-21281.

6. Thompson VC, Day TK, Bianco-Miotto T, Selth LA, Han G, Thomas M, Buchanan G, Scher HI, Nelson CC, Greenberg NM, Butler LM and Tilley WD. A gene signature identified using a mouse model of androgen receptor-dependent prostate cancer predicts biochemical relapse in human disease. Int J Cancer. 2012; 131:662-672. doi: 610.1002/ijc.26414. Epub 22012 Jan 26424.

7. Rubin MA, Zhou M, Dhanasekaran SM, Varambally S, Barrette TR, Sanda MG, Pienta KJ, Ghosh D and Chinnaiyan AM. alpha-Methylacyl coenzyme A racemase as a tissue biomarker for prostate cancer. JAMA. 2002; 287:1662-1670.

8. Vaarala MH, Hirvikoski P, Kauppila S and Paavonen TK. Identification of androgen-regulated genes in human prostate. Mol Med Report. 2012; 6:466-472.

9. Sartori DA and Chan DW. Biomarkers in prostate cancer: what's new? Current opinion in oncology. 2014; 26:259- 
264.

10. Bishoff JT, Freedland SJ, Gerber L, Tennstedt P, Reid J, Welbourn W, Graefen M, Sangale Z, Tikishvili E, Park J, Younus A, Gutin A, Lanchbury JS, Sauter G, Brawer M, Stone S, et al. Prognostic utility of the cell cycle progression score generated from biopsy in men treated with prostatectomy. The Journal of urology. 2014; 192:409414.

11. Klein EA, Cooperberg MR, Magi-Galluzzi C, Simko JP, Falzarano SM, Maddala T, Chan JM, Li J, Cowan JE, Tsiatis AC, Cherbavaz DB, Pelham RJ, Tenggara-Hunter I, Baehner FL, Knezevic D, Febbo PG, et al. A 17-gene Assay to Predict Prostate Cancer Aggressiveness in the Context of Gleason Grade Heterogeneity, Tumor Multifocality, and Biopsy Undersampling. European urology. 2014.

12. Nguyen HG, Welty CJ and Cooperberg MR. Diagnostic associations of gene expression signatures in prostate cancer tissue. Current opinion in urology. 2015; 25:65-70.

13. Johnson IR, Parkinson-Lawrence EJ, Butler LM and Brooks DA. Prostate cell lines as models for biomarker discovery: Performance of current markers and the search for new biomarkers. The Prostate. 2014; 74:547-560.

14. Johnson IR, Parkinson-Lawrence EJ, Shandala T, Weigert R, Butler LM and Brooks DA. Altered endosome biogenesis in prostate cancer has biomarker potential. Molecular cancer research : MCR. 2014; 12:1851-1862.

15. Kallunki T, Olsen OD and Jaattela M. Cancer-associated lysosomal changes: friends or foes? Oncogene. 2012.

16. Nomura $\mathrm{T}$ and Katunuma N. Involvement of cathepsins in the invasion, metastasis and proliferation of cancer cells. J Med Invest. 2005; 52:1-9.

17. Tomlins SA, Mehra R, Rhodes DR, Cao X, Wang L, Dhanasekaran SM, Kalyana-Sundaram S, Wei JT, Rubin MA, Pienta KJ, Shah RB and Chinnaiyan AM. Integrative molecular concept modeling of prostate cancer progression. Nat Genet. 2007; 39:41-51.

18. Glinsky GV, Glinskii AB, Stephenson AJ, Hoffman RM and Gerald WL. Gene expression profiling predicts clinical outcome of prostate cancer. The Journal of clinical investigation. 2004; 113:913-923.

19. de Hoon MJ, Imoto S, Nolan J and Miyano S. Open source clustering software. Bioinformatics. 2004; 20:1453-1454.

20. Parkin DM, Bray F, Ferlay J and Pisani P. Global cancer statistics, 2002. CA Cancer J Clin. 2005; 55:74-108.

21. AACR A. (2012). Cancer in Australia: an overview 2012. Cancer series no. 74. Cat. no. CAN 70. Canberra: AIHW.

22. Schroder FH, Hugosson J, Roobol MJ, Tammela TL, Ciatto S, Nelen V, Kwiatkowski M, Lujan M, Lilja H, Zappa M, Denis LJ, Recker F, Berenguer A, Maattanen L, Bangma $\mathrm{CH}$, Aus $\mathrm{G}$, et al. Screening and prostate-cancer mortality in a randomized European study. The New England journal of medicine. 2009; 360:1320-1328.

23. Stephenson AJ, Scardino PT, Eastham JA, Bianco FJ, Jr., Dotan ZA, DiBlasio CJ, Reuther A, Klein EA and
Kattan MW. Postoperative nomogram predicting the 10year probability of prostate cancer recurrence after radical prostatectomy. Journal of clinical oncology : official journal of the American Society of Clinical Oncology. 2005; 23:7005-7012.

24. Murphy L, Prencipe M, Gallagher WM and Watson RW. Commercialized biomarkers: new horizons in prostate cancer diagnostics. Expert Rev Mol Diagn. 2015; 15:491503.

25. Crawford ED, Rove KO, Trabulsi EJ, Qian J, Drewnowska KP, Kaminetsky JC, Huisman TK, Bilowus ML, Freedman SJ, Glover WL, Jr. and Bostwick DG. Diagnostic performance of PCA3 to detect prostate cancer in men with increased prostate specific antigen: a prospective study of 1,962 cases. The Journal of urology. 2012; 188:1726-1731.

26. Ferreira LB, Palumbo A, de Mello KD, Sternberg C, Caetano MS, de Oliveira FL, Neves AF, Nasciutti LE, Goulart LR and Gimba ER. PCA3 noncoding RNA is involved in the control of prostate-cancer cell survival and modulates androgen receptor signaling. BMC Cancer. 2012; 12:507.

27. Ruckhaberle E, Holtrich U, Engels K, Hanker L, Gatje R, Metzler D, Karn T, Kaufmann M and Rody A. Acid ceramidase 1 expression correlates with a better prognosis in ER-positive breast cancer. Climacteric. 2009; 12:502513.

28. Seelan RS, Qian C, Yokomizo A, Bostwick DG, Smith DI and Liu W. Human acid ceramidase is overexpressed but not mutated in prostate cancer. Genes Chromosomes Cancer. 2000; 29:137-146.

29. Dong JT. Chromosomal deletions and tumor suppressor genes in prostate cancer. Cancer Metastasis Rev. 2001; 20:173-193.

30. Kela I, Harmelin A, Waks T, Orr-Urtreger A, Domany E and Eshhar Z. Interspecies comparison of prostate cancer gene-expression profiles reveals genes associated with aggressive tumors. Prostate. 2009; 69:1034-1044.

31. Liong ML, Lim CR, Yang $\mathrm{H}$, Chao $\mathrm{S}$, Bong $\mathrm{CW}$, Leong WS, Das PK, Loh CS, Lau BE, Yu CG, Ooi EJ, Nam RK, Allen PD, Steele GS, Wassmann K, Richie JP, et al. Bloodbased biomarkers of aggressive prostate cancer. PLoS ONE. 2012; 7:e45802.

32. March-Villalba JA, Martinez-Jabaloyas JM, Herrero MJ, Santamaria J, Alino SF and Dasi F. Cell-free circulating plasma hTERT mRNA is a useful marker for prostate cancer diagnosis and is associated with poor prognosis tumor characteristics. PLoS ONE. 2012; 7:e43470.

33. Pelosi G, Schianchi E, Dell'orto P, Veronesi G, Spaggiari L, Pasini F, Sozzi G, Brambilla E, Griso C and Viale G. Detecting cell-free circulating hTERT mRNA in the plasma may identify a subset of nonsmall cell lung cancer patients. Virchows Arch. 2006; 448:7-15.

34. Rasiah KK, Stricker PD, Haynes AM, Delprado W, Turner JJ, Golovsky D, Brenner PC, Kooner R, O’Neill GF, 
Grygiel JJ, Sutherland RL and Henshall SM. Prognostic significance of Gleason pattern in patients with Gleason score 7 prostate carcinoma. Cancer. 2003; 98:2560-2565.

35. Peshavariya H, Dusting GJ, Jiang F, Halmos LR, Sobey CG, Drummond GR and Selemidis S. NADPH oxidase isoform selective regulation of endothelial cell proliferation and survival. Naunyn Schmiedebergs Arch Pharmacol. 2009; 380:193-204.

36. Vickers AJ, Roobol MJ and Lilja H. Screening for prostate cancer: early detection or overdetection? Annu Rev Med. 2012; 63:161-170.

37. Aizer AA, Chen MH, Hattangadi J and D'Amico AV. Initial management of prostate-specific antigen-detected, low-risk prostate cancer and the risk of death from prostate cancer. BJU Int. 2013. 